\title{
Evolución de la tasa de filtración glomerular y proteinuria a largo plazo en niños monorrenos en un hospital nacional
}

\author{
Long-term evolution of glomerular filtration rate and proteinuria in single kidney children in a national \\ hospital
}

Ángel Eduardo Villarreal Giraldo 1,a, Renato Andrés Ferrándiz Espadín 1,a, Carlos Eduardo Salinas Cier 1,a, César Antonio Loza Munarriz 1,d; 2,b, Juan José García Bustinza 1,e; 3,c, Walter Augusto Higueras Herrera ${ }^{3, c}$

\section{RESUMEN}

Objetivos: Determinar la evolución de la tasa de filtración glomerular (TFG) y los niveles de proteinuria en 24 horas en pacientes monorrenos con un tiempo mínimo de seguimiento de 5 años. Material y métodos: Se utilizaron los registros de los pacientes monorrenos atendidos en el Hospital Nacional Edgardo Rebagliati Martins (HNERM) entre 2005-2016. Se consignaron los datos de proteinuria en 24 horas y tasa de filtración glomerular en orina de 24 horas de 50 pacientes que cumplieron los criterios de inclusión del estudio. Resultados: La mediana de la TFG de los 50 pacientes fue 109,74 ml/min/1,73m² (RIQ 86,1-138,75). Hubo 22 pacientes en estadio I, 2 en estadio II y 5 en estadio III de acuerdo a la clasificación de enfermedad renal crónica (ERC). Se encontró 26 (52\%) pacientes con proteinuria significativa y persistente, no hubo en rango nefrótico. Conclusiones: Los pacientes monorrenos presentan a largo plazo alteraciones como hiperfiltración y disminución de la TFG.

PALABRAS CLAVE: Riñón único, proteinuria, tasa de filtración glomerular. (Fuente: DeCS BIREME).

\section{SUMMARY}

Objectives: To determine the glomerular filtration rate (GFR) and 24-hour proteinuria among single kidney patients with a minimum 5-year follow-up period. Methods: The medical charts of patients attended at Hospital Nacional Edgardo Rebagliati Martins (HNERM) from 2005-2016 were review. Information on 50 patients in whom data on GFR and 24-hour proteinuria was analyzed. Results: Median GFR was $109.74 \mathrm{ml} / \mathrm{min} / 1.73 \mathrm{~m} 2$ (RIQ 86.1-138.75); 22 patients were in the chronic renal disease stage classification I, 2 in chronic renal disease stage classification II and 5 in stage III; 26 patients (52\%) had persistent and significant proteinuria, none of these had proteinuria in the nephrotic range. Conclusions: Single kidney patients present long-term complications such as hyperfiltration and reduction of the GFR.

KEYWORDS: Solitary kidney, proteinuria, glomerular filtration rate. (Source: MeSH NLM).

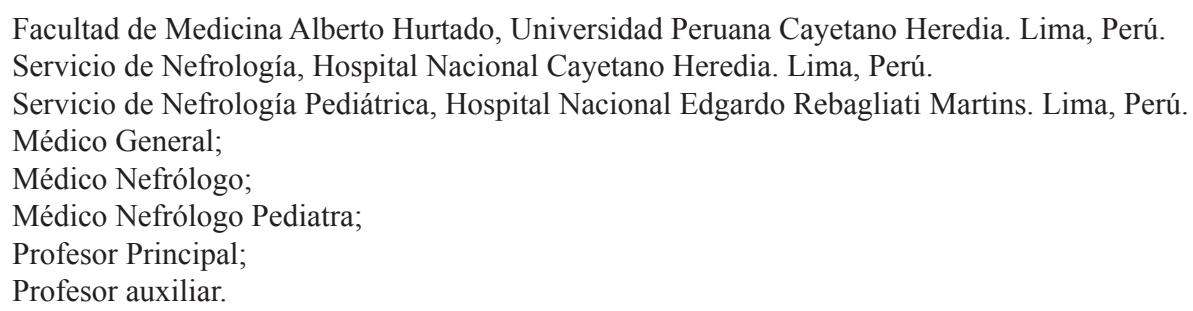




\section{INTRODUCCIÓN}

El riñón solitario funcionante es una condición que ocurre por ausencia anatómica o funcional de un riñón, compensando el riñón solitario, la función de ambos (1). Estos pacientes tienen un menor número de nefronas para mantener la misma tasa de filtración glomerular (TFG), lo que los predispone a desarrollar alteración en la función renal (2), pudiéndose expresar como deterioro de la misma, presión arterial (PA) elevada o proteinuria. Se subdividen en monorrenos reales, aquellos con ausencia anatómica de un riñón (nefrectomía y agenesia renal unilateral) y, funcionales, aquellos con presencia de ambos riñones, pero solo uno funcionante y el otro con anormalidades que tienden a involucionar (aplasia renal y displasia renal multiquística).

Dentro de los monorrenos reales, la agenesia renal unilateral se define como el fallo en el inicio de la secuencia pronefros, mesonefros (formación de la yema ureteral) y metanefros (formación del blastema metanéfrico que origina las nefronas) $(3,4)$. Nefrectomizado, es el paciente sometido a la extracción quirúrgica de una parte o todo el riñón (5).

Dentro de los monorrenos funcionales, aquellos con aplasia renal poseen un uréter y un riñón rudimentario sin nefronas funcionales; esto puede resultar de la temprana regresión de la yema ureteral, de una diferencia metanéfrica alterada o por defectos en la formación de los túbulos colectores (6). Los pacientes con displasia renal multiquística unilateral (DRMU) presentan una alteración que produce estructuras que no poseen función; como cartílago, tejido fibroso y tejido epitelial anormal, y presentan ausencia gammagráfica $(7,8)$. Finalmente, es necesario definir la hipoplasia renal, pues se confunde con aplasia renal y DRMU. Esta se define como un riñón anormalmente pequeño, no displásico, menor de $2 \mathrm{DE}$ por debajo de la media que correlaciona con la edad y los parámetros de crecimiento (9), tiene un menor número de cálices y nefronas y puede o no tener función; por lo que no se incluye en el presente estudio.

Se han descrito varios estudios que analizan a los diversos tipos de pacientes monorrenos y los cambios que presentan en la tasa de filtración glomerular y otros parámetros como la proteinuria, microalbuminuria e hipertensión arterial (HTA) (10-14). En estos estudios se evidencian seguimientos irregulares de los pacientes y la ausencia de evaluaciones rigurosas de la condición inicial de los mismos; lo que podría subestimar el verdadero impacto de ser monorreno.
El objetivo del estudio fue determinar la evolución de la tasa de filtración glomerular y los niveles de proteinuria en 24 horas, en pacientes monorrenos con un tiempo mínimo de seguimiento de 5 años.

\section{MATERIAL Y MÉTODOS}

Estudio tipo serie de casos, descriptivo. Se revisaron las historias clínicas de pacientes monorrenos atendidos durante el periodo 2005-2016 en el servicio de Nefrología Pediátrica del Hospital Nacional Edgardo Rebagliati Martins (HNERM) con un tiempo de seguimiento mínimo de 5 años desde su diagnóstico.

La muestra constituyó pacientes menores de 14 años, que cumplían con los siguientes criterios de inclusión: Pacientes monorrenos funcionales y reales con al menos 5 años de seguimiento, diagnosticados por gammagrafía renal donde se demuestre ausencia de un riñón y presencia del riñón contralateral funcionante. En los pacientes monorrenos reales nefrectomizados, se consideraron aquellos sometidos a la extracción total de un riñón, debiendo contar con por lo menos una tomografía y un control de creatinina sérica previo a la nefrectomía, para hacer el cálculo de la TFG con la fórmula de Schwartz e identificar si existía compromiso del riñón no afectado previo a la cirugía, considerando dentro del estudio solo aquellos con una TFG calculada normal.

Se excluyeron pacientes con otra enfermedad renal (infección del tracto urinario recurrente, síndrome nefrótico, vejiga neurogénica, etc.), con alteración en su único riñón funcionante (hipoplasia o zonas de displasia en su único riñón funcionante) y a los que tuviesen enfermedad renal crónica (ERC) terminal o el antecedente de haber sido trasplantado.

\section{Definición operacional de las variables}

Las variables del estudio fueron: La TFG y la proteinuria en orina de 24 horas. Además, se evaluaron la PA, densidad urinaria e índice de masa corporal (IMC). La TFG se determinó por la depuración de creatinina en $\mathrm{ml} / \mathrm{min} / 1,73 \mathrm{~m}^{2}$, la determinación de la creatinina se realizó por el método de Jaffé (utilizado en todos los pacientes del servicio de Nefrología Pediátrica en el laboratorio del HNERM) (15-17).

Se clasificó la ERC según la versión adaptada a población pediátrica del Kidney Disease Outcomes Quality Initiative (K/DOQI) $(18,19)$. La proteinuria se clasificó en: Normal $<4 \mathrm{mg} / \mathrm{m}^{2} / \mathrm{h}$, proteinuria 
significativa $>4 \mathrm{mg} / \mathrm{m}^{2} / \mathrm{h} \mathrm{y}<40 \mathrm{mg} / \mathrm{m}^{2} / \mathrm{h}$ y, proteinuria en rangos nefróticos $>40 \mathrm{mg} / \mathrm{m}^{2} / \mathrm{h}$. La proteinuria persistente se definió como la presencia de proteinuria significativa en más de 2 controles $(20,21)$.

\section{Procedimientos del estudio}

Se revisaron 378 historias clínicas, de las cuales solo 243 se encontraron completas, obteniendo finalmente 50 pacientes que cumplían los criterios de inclusión (gráfico 1).

Para el análisis de los datos, las variables categóricas fueron expresadas en tasas y proporciones y las variables continuas como medias y desviación estándar o medianas y rango intercuartil, dependiendo del tipo de distribución de la variable.

Para comparar variables y evaluar relación en forma exploratoria, se utilizó la prueba de chi cuadrado en las variables categóricas y en las variables continuas, se usó la prueba de suma de Rangos de Wilcoxon, T test y ANOVA, dependiendo del tipo de distribución. Se exploró la correlación entre los niveles de proteinuria en orina de 24 horas y la TFG usando la Prueba de rangos de Spearman (rs).

La información fue registrada en Microsoft Excel y luego fue importada a STATA v. 14 para su análisis. Se consideró $\mathrm{p} \leq 0,05$ como estadísticamente significativo.

\section{Aspectos éticos}

Los datos proporcionados por el HNERM se manejaron de manera confidencial solo por los investigadores. La base de datos fue codificada, sin incluir la identidad de los pacientes. El estudio contó con la aprobación de los comités de ética del HNERM y la Universidad Peruana Cayetano Heredia (UPCH).

\section{RESULTADOS}

De los 50 pacientes del estudio, 26 (52\%) fueron mujeres. El rango de edad de los pacientes fue de 5-13,5 años. Veinticinco (50\%) pacientes eran monorrenos funcionales y $25(50 \%)$ reales. Dentro del grupo de monorrenos funcionales solo se encontraron

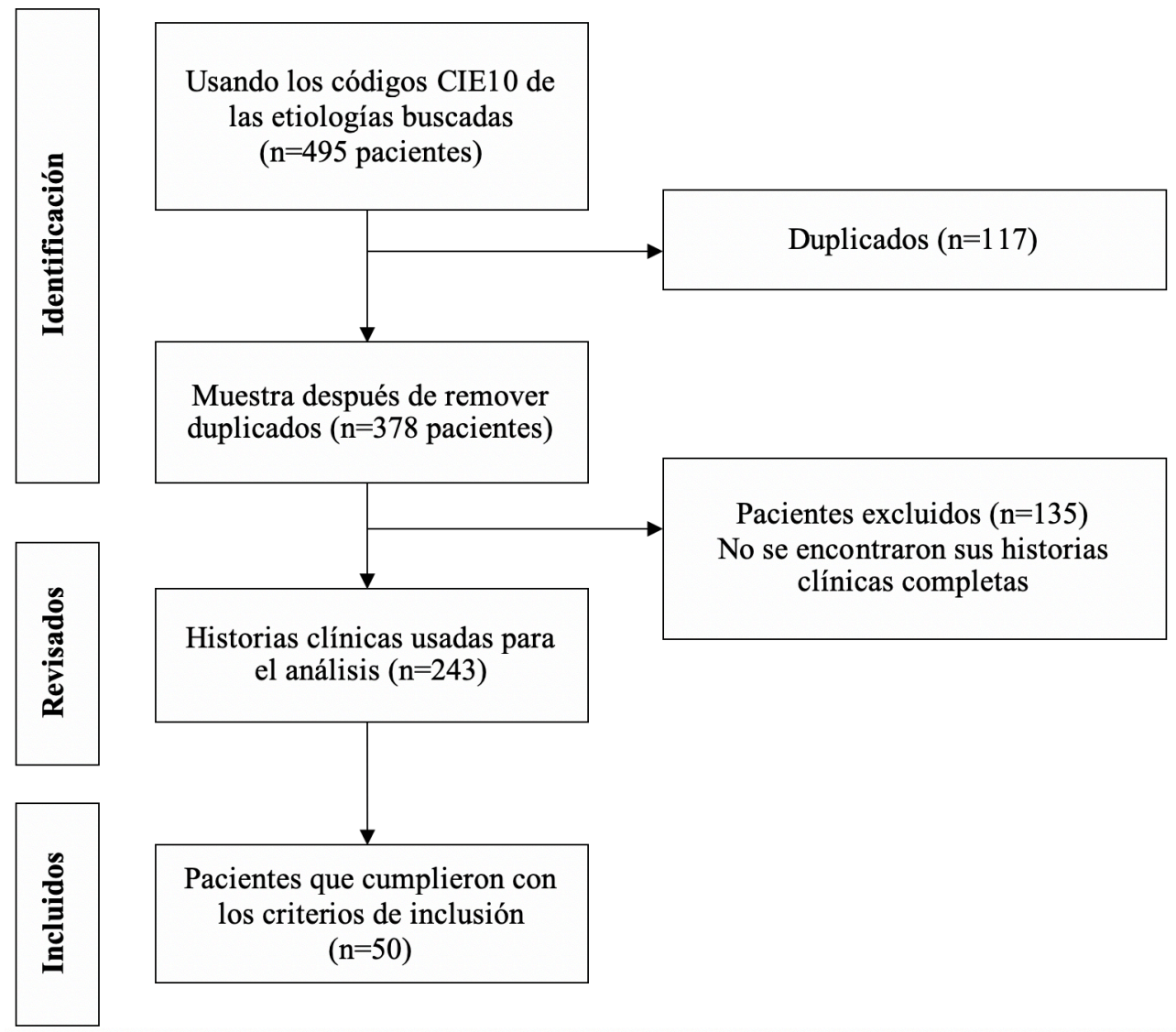

Gráfico 1. Proceso de selección de pacientes incluidos en el estudio. 
Tabla 1. Relación entre tipo de monorreno y estadio de enfermedad renal.

\begin{tabular}{lcccc}
\hline Etiología & Sin Daño Renal & Estadio I & Estadio II & Estadio III \\
\hline Nefrectomizado Unilateral & 2 & 5 & 0 & 2 \\
DRMU & 11 & 10 & 1 & 3 \\
Agenesia Renal Unilateral & 8 & 7 & 1 & 0 \\
TOTAL & 21 & 22 & 2 & 5 \\
\hline
\end{tabular}

DRMU: Displasia renal multiquística unilateral

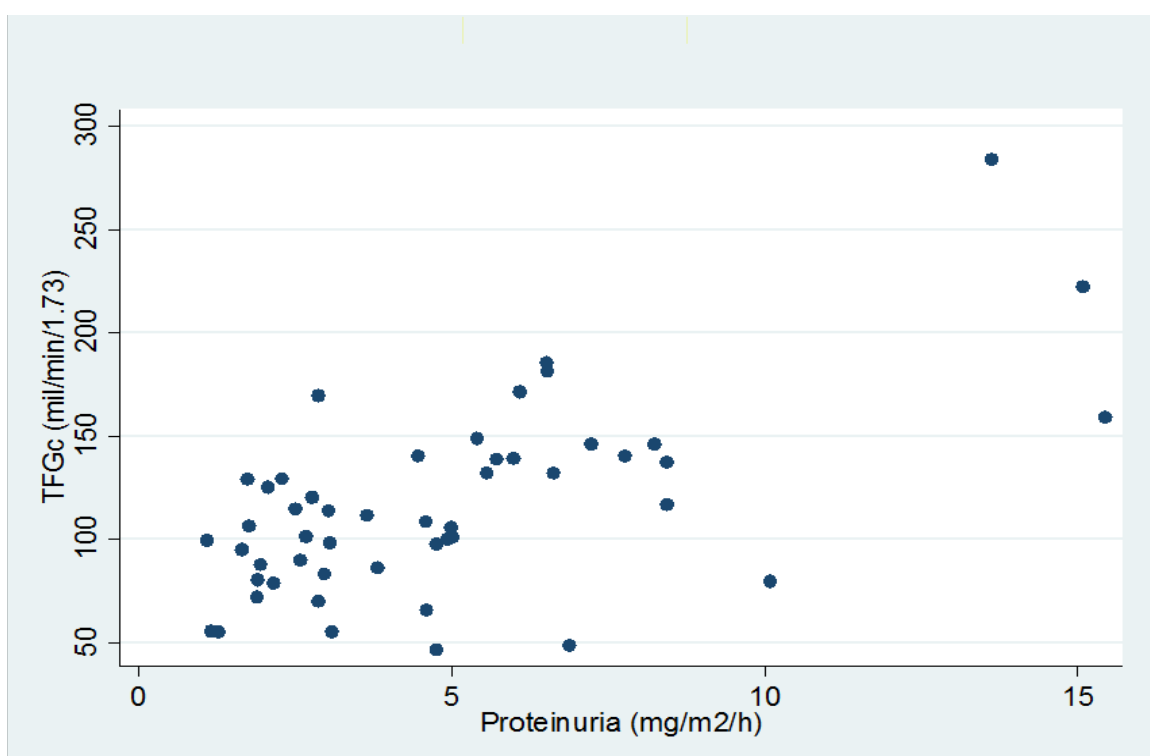

Gráfico 2. Correlación entre los niveles de la tasa de filtración glomerular (TFG) y los niveles de proteinuria.

pacientes con DRMU. En el grupo de monorrenos reales, hubo $16(32 \%)$ con agenesia renal y $9(18 \%)$ que fueron sometidos a nefrectomía. En $32(64 \%)$ pacientes el diagnóstico fue prenatal y en $18(36 \%)$, postnatal (mediana de 2 años; RIQ 2-4 años). La mediana del tiempo de seguimiento de los pacientes desde su diagnóstico fue 5,95; RIQ de 5,4-7.

De los 9 (18\%) pacientes con nefrectomía, 7 (14\%) fueron operados por Tumor de Wilms, 1 (2\%) por estenosis de la arteria renal y $1(2 \%)$ por hidronefrosis. La edad a la que fueron operados tuvo una mediana de dos años (RIQ 1-4 años). Tres pacientes con diagnóstico inicial de DRMU fueron sometidos a nefrectomía aproximadamente al año de edad; sin embargo, se les consideró dentro del grupo de pacientes con DRMU.

La mediana de la TFG de los 50 pacientes fue $109,74 \mathrm{ml} / \mathrm{min} / 1,73 \mathrm{~m}^{2}$ (RIQ $86,1-138,75$ ). El grupo con agenesia renal tuvo una media de TFG de 103,73 $\pm 28,99 \mathrm{ml} / \mathrm{min} / 1,73 \mathrm{~m}^{2}$ y el grupo de nefrectomizados, $116,31 \pm 44,28 \mathrm{ml} / \mathrm{min} / 1,73 \mathrm{~m}^{2}$. El grupo de monorrenos funcionales, una mediana de $113,6 \mathrm{ml} /$ $\mathrm{min} / 1,73 \mathrm{~m}^{2}$ (RIQ 94,9-140).

Se encontraron 26 (52\%) pacientes con proteinuria significativa y persistente, no hubo en rango nefrótico (mediana de $6,3 \mathrm{mg} / \mathrm{m}^{2} / \mathrm{h}$; RIQ 4,99-8,24); de ellos, $12(24 \%)$ fueron monorrenos funcionales (mediana de $7,2 \mathrm{mg} / \mathrm{m}^{2} / \mathrm{h}$; RIQ $\left.5,36-11,86\right)$ y $14(28 \%)$, reales ( 8 con agenesia renal, media de $5,93 \pm 1,62 \mathrm{mg} / \mathrm{m}^{2} / \mathrm{h}$ y 6 con nefrectomía, media de $6,16 \pm 0,88 \mathrm{mg} / \mathrm{m}^{2} / \mathrm{h}$ ). No se encontró diferencia estadística en los niveles de proteinuria según la etiología $(\mathrm{p}=0,61)$.

En la tabla 1 se muestra el estadio de enfermedad renal según el tipo etiológico de monorreno. Se encontraron 29 pacientes con ERC: 2 en estadio II y 5 en estadio III. No se encontró diferencias en la TFG según la etiología $(\mathrm{p}=0,39)$.

Se encontró correlación entre la TFG y la proteinuria $(\mathrm{rs}=0,52 ; \mathrm{p}=0,0001) \quad$ (gráfico 2). La correlación entre la TFG y la proteinuria en los pacientes con 


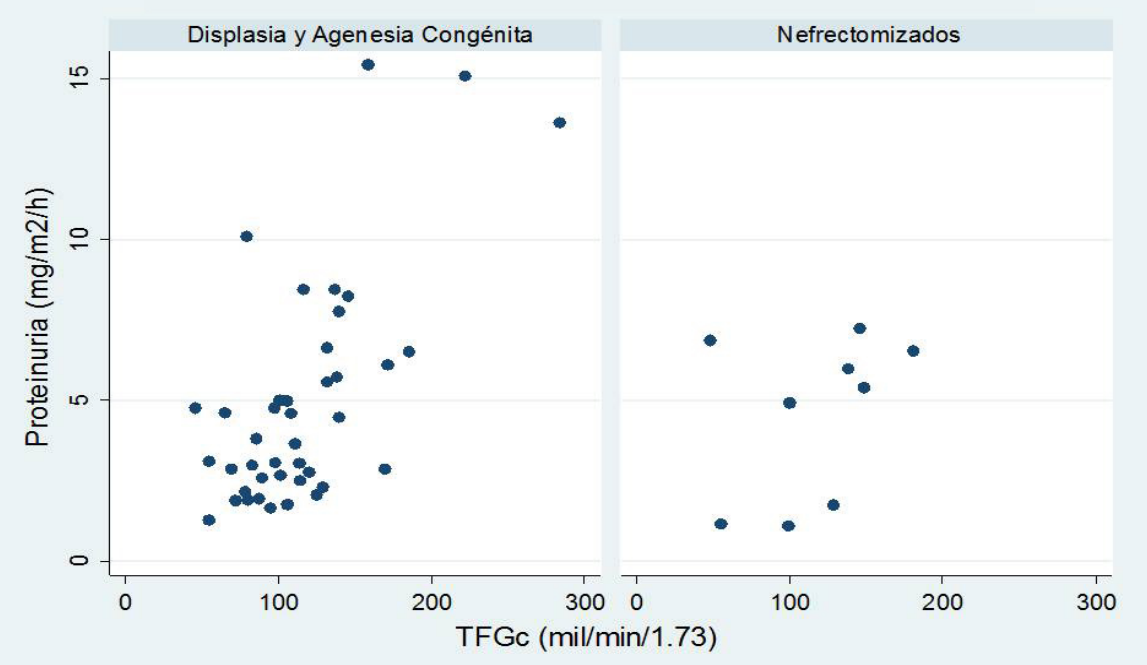

Gráfico 3. Correlación entre los niveles de la tasa de filtración glomerular y los niveles de proteinuria, según la etiología.

displasia y agenesia fue $r s=0,53 \quad(p=0,0003)$. En los nefrectomizados no hubo correlación significativa $(\mathrm{rs}=0,38 ; \mathrm{p}=0,30)$ (gráfico 3). Finalmente, al comparar los niveles de proteinuria entre pacientes con agenesia y displasia y pacientes nefrectomizados, no se encontró diferencia estadística $(\mathrm{p}=0,98)$.

\section{DISCUSIÓN}

Este estudio es el primero en analizar a los pacientes monorrenos con un tiempo de seguimiento definido; además que la TFG usada para el análisis fue hallada con depuración de creatinina en orina de 24 horas. En este estudio se usó la clasificación de severidad de daño renal adaptada a la población pediátrica del Kidney Disease Outcomes Quality Initiative (KDOQI) (18). Si bien existe una versión actualizada de esta guía, Kidney Disease: Improving Global Outcomes (KDIGO) (22), no está adaptada a niños. Ningún otro estudio ha usado estas clasificaciones en pacientes monorrenos.

Comparando con otros estudios, Baudoin et al. (10), en 1993, en pacientes nefrectomizados $(n=111)$, encontraron una media de TFG de $86 \pm 17 \mathrm{ml} /$ $\mathrm{min} / 1,73 \mathrm{~m}^{2} ; 48$ pacientes con TFG entre $90-60 \mathrm{ml} /$ $\mathrm{min} / 1,73 \mathrm{~m}^{2}$ y 3 pacientes con $\mathrm{TFG}<60 \mathrm{ml} / \mathrm{min} / 1,73 \mathrm{~m}^{2}$. Es importante mencionar que en ese estudio solo se midió la proteinuria una sola vez. Robitaille et al. (11) en 1985, en 27 pacientes pediátricos nefrectomizados (elegidos mediante un pielograma), encontraron una media de TFG similar a la encontrada por Baudoin et al. Si bien los resultados de nuestro estudio difieren de los estudios citados, en dichos trabajos no se precisó el estado funcional inicial del riñón; los pacientes monorrenos requieren un estudio gammagráfico al inicio de su seguimiento o un estudio de creatinina sérica para asegurar una función renal normal antes de su cirugía.

En el estudio de Baudoin et al. (10), se encontró entre las causas principales de nefrectomía: 41 por uropatía obstructiva, 34 por reflujo, 14 por tumor de Wilms y 11 por cálculos. En el estudio de Robitaille et al. (11), se observó que las principales causas de nefrectomía eran la hidronefrosis, seguida del tumor de Wilms. A diferencia de los estudios anteriores, en nuestro estudio, la principal causa de nefrectomía fue tumor de Wilms, seguido de nefromegalia y estenosis de la arteria renal; esto puede deberse a que los pacientes con problemas obstructivos tienen mayor riesgo de presentar infecciones urinarias a repetición, lo que condiciona la aparición de cicatrices y la alteración de su función renal, por lo que no serían aptos de ser incluidos dentro de la muestra de nuestro estudio.

En cuanto a la agenesia renal unilateral, en el metaanálisis realizado por Westland et al. (12), el promedio de seguimiento de los pacientes pediátricos fue de 9,1 años y de los 43 estudios que analizaron, en 11 tenían un promedio de TFG en $100 \mathrm{ml} / \mathrm{min} / 1,73 \mathrm{~m}^{2}$, similar al encontrado en nuestro estudio. De los 575 pacientes con los que contaba ese estudio, 18,8\% tenía 
proteinuria y el 9,9\% tenía TFG $<60 \mathrm{ml} / \mathrm{min} / 1,73 \mathrm{~m}^{2}$. En nuestro estudio, el 16\% de pacientes con agenesia renal tuvo una media de proteinuria de $5,93 \pm 1,62 \mathrm{mg} /$ $\mathrm{m}^{2} / \mathrm{h}$.

Por otro lado, Weinstein et al. (14), estudiaron a 80 pacientes con DRMU, pero sin una evaluación funcional del riñón remanente (solo se realizó ecografía); la media de seguimiento fue de 5,3 años $(0,25-15,3)$; la TFG media (estimada por fórmula de Schwartz) fue de $108,9 \mathrm{ml} / \mathrm{min} / 1,73 \mathrm{~m}^{2}$ y 3 pacientes presentaron una TFG $<70 \mathrm{ml} / \mathrm{min} / 1,73 \mathrm{~m}^{2} ;$ no obstante, estos pacientes tenían menos de 8 meses de edad. La madurez del riñón se completa a los 2 años de edad (23), por lo que no resulta apropiado evaluar la función de un monorreno menor de 2 años porque se estaría subestimando su TFG. Además, debido a que su población era seguida por diferentes especialidades (Pediatría, Urología y Nefrología Pediátrica), sin controles regulares, el trabajo se enfocó en la regresión ecográfica del riñón displásico y no hizo énfasis en la evolución de la función renal remanente en estos pacientes.

Aslam et al. (24), en un estudio prospectivo en pacientes monorrenos pediátricos con DRMU, reportaron que 31 pacientes que se controlaron hasta los 10 años mantuvieron una TFG promedio de 86,1 $\mathrm{ml} / \mathrm{min} / 1,73 \mathrm{~m}^{2}$, de los cuales 16 tenían TFG entre $60-90 \mathrm{ml} / \mathrm{min} / 1,73 \mathrm{~m}^{2}$ y 2 pacientes, TFG $<60 \mathrm{ml} /$ $\mathrm{min} / 1,73 \mathrm{~m}^{2}$. Sin embargo, no se especifica cómo se obtuvo la TFG, además no se midió proteinuria ni la presión arterial. Y aunque se realizó gammagrafía con DMSA marcado con Tc99 a todos los pacientes a los tres meses de haberse realizado el diagnóstico, se incluyó a pacientes con reflujo vésico-ureteral. Para los pacientes con DRMU de nuestro estudio, los datos no tenían distribución normal por lo que las medidas de tendencia central no son comparables con las de los otros trabajos.

Es importante indicar que, aunque en nuestro estudio se usó la mediana de la TFG, si se hubiese calculado la media de la TFG como en los estudios citados, también se observaría un valor de TFG dentro de rangos normales $\left(115,90 \pm 45,13 \mathrm{ml} / \mathrm{min} / 1,73 \mathrm{~m}^{2}\right)$; lo que implica que se está subestimando el valor real de la TFG de algunos pacientes. Esto sugiere que la función renal debe ser clasificada por estadiaje y no por promedios de TFG.

También se encontró que en pacientes con DRMU y agenesia renal, los niveles elevados de
TFG se correlacionan con proteinuria significativa y persistente, lo que no se observa en los pacientes nefrectomizados. Esto es importante, ya que la hiperfiltración que presentan de forma compensatoria estos pacientes monorrenos puede ser un factor de riesgo para presentar ERC (25) y; por lo tanto, deben tener un seguimiento continuo.

No se pudo determinar con exactitud el diagnóstico de todos los pacientes con agenesia renal porque no todos contaron con una ecografía prenatal que descarte la presencia de un riñón aplásico involucionado. Asimismo, no se tuvo una población mayor de pacientes con agenesia renal, puesto que un porcentaje significativo de este tipo de pacientes presenta anormalidades en las vías urinarias lo que conlleva a deterioro de la función renal.

En las definiciones actuales de ERC no se considera el ser monorreno como daño renal, por lo que se requieren estudios prospectivos con una metodología rigurosa para determinar si estos pacientes entran en esta categoría sin la necesidad de presentar HTA o proteinuria asociadas.

Como conclusión del estudio se puede decir que los pacientes monorrenos, a largo plazo, pueden presentar alteración en la función renal, como hiperfiltración y disminución de la TFG. En nuestro estudio, en casi la mitad de los casos se encontró proteinuria significativa y persistente e hiperfiltración; e incluso hubo algunos pacientes con daño renal en estadio II y III. Se correlacionó positivamente la presencia de proteinuria e incremento de la TFG; sin embargo, no necesariamente implica hiperfiltración. Se deben hacer más estudios en el futuro para poder usarlo como marcador en estos pacientes.

\section{Agradecimientos:}

Al Instituto Nacional de Salud del Niño - San Borja, como auspiciador de esta investigación.

\section{Declaración de financiamiento y de conflictos de interés:}

El estudio fue financiado por los autores. Los autores declaran no tener conflictos de intereses.

\section{Contribución de autoría:}

AEVG: Participó en el diseño del estudio y en la redacción del artículo. RAFE: Participó en el 
diseño del estudio y en la redacción del artículo. CESC: Participó en la recolección de los datos y en la redacción del artículo. CALM: Participó en la interpretación de los resultados. JJGB: Participó en el diseño del estudio y en la redacción del artículo.

WAHH: Participó en el diseño del estudio.

\section{Correspondencia:}

Carlos Eduardo Salinas Cier

Correo electrónico: carlos.salinas.c@upch.pe

\section{REFERENCIAS BIBLIOGRÁFICAS}

1. Carter C, Evans K, Pescia G. A family study of renal agenesis. J Med Genet. 1979; 16:176-188.

2. Brenner BM, Lawler EV, Mackenzie HS. The hyperfiltration theory: a paradigm shift in nephrology. Kidney Int. 1996; 49 (6):1774-1777.

3. Yalavarthy R, Parikh CR. Congenital renal agenesis: A review. Saudi J Kidney Dis Transpl. 2003; 14:336341.

4. Sarhan OM, Albedaiwi K. Unilateral renal agenesis: Necessity of postnatal evaluation in a contemporary series. Eur Urol Suppl. 2016; 15(3):701.

5. Kenney PA, Wotkowicz T, Libertino JA. Contemporary open surgery of the kidney. In: Wein AJ, ed. Campbell-Walsh Urology. Philadelphia, Pa: Saunders Elsevier; 2011.

6. Hiraoka M, Tsukahara H, Ohshima Y, Kasuga K, Ishihara Y, Mayumi M. Renal aplasia is the predominant cause of congenital solitary kidneys. Kidney Int. 2002; 61:1840-1844.

7. Greenbaum L. Renal dysplasia and MRI: a clinician's perspective. Pediatr Radiol. 2008; 38(Suppl1):S70 S75.

8. Hains $\mathrm{D}$, Bates $\mathrm{C}$, Ingraham $\mathrm{S}$, Schwaderer A. Management and etiology of the unilateral multicystic dysplastic kidney: a review. Pediatr Nephrol. 2009; 24:233-241.

9. Cain JE, Di Giovanni V, Smeeton J, Rosenblum ND. Genetics of renal hypoplasia: Insights into the Mechanisms Controlling Nephron Endowment. Pediatr Res. 2010; 68:91-98.

10. Baudoin P, Provoost AP, Molenaar JC. Renal function up to 50 years after unilateral nephrectomy in childhood. Am J Kidney Dis. 1993; 21:603-611.

11. Robitaille P, Mongeau JG, Lortie L, Sinnassamy P. Long-term follow-up of patients who underwent unilateral nephrectomy in childhood. Lancet. 1985; 1(8441):1297-1299.

12. Westland R, Schreuder MF, Ket JC, van Wijk JA. Unilateral renal agenesis: a systematic review on associated anomalies and renal injury. Nephrol Dial Transplant. 2013; 28:1844-1855.
13. Narchi H. Risk of hypertension with multicystic kidney disease: a systematic review. Arch Dis Child. 2005; 90:921-924.

14. Weinstein A, Goodman TR, Iragorri S. Simple multicystic dysplastic kidney disease: End points for subspecialty follow-up. Pediatr Nephrol. 2008; 23:111-116.

15. Rodríguez N, Torres D, Carvajal M. Confiabilidad del Método de Jaffé modificado por Laboratorios Heiga para la determinación automatizada de la Creatinina. Rev Fac Farm. 2001; 42:55-57.

16. Perazzi B, Angerosa M. Creatinina en sangre: calidad analítica e influencia en la estimación del Índice de Filtrado Glomerular. Acta Bioquím Clín Latinoam. 2011; 45(2):265-272.

17. Peake M, Whiting M. Measurement of serum creatinine-current status and future goals. Clin Biochem Rev. 2006; 27(4):173-84.

18. Hogg RJ, Furth S, Lemley KV, et al. National Kidney Foundation's Kidney Disease Outcomes Quality Initiative clinical practice guidelines for chronic kidney disease in children and adolescents: Evaluation, classification, and stratification. Pediatrics. 2003; 111:1416-1421.

19. Johnson D. Diagnosis, classification and staging of chronic kidney disease. Sidney: Kidney Health Australia-caring for Australians with renal impairments (KHA-CARI); 2012. p. 1-31.

20. Miltényi M. Urinary protein excretion in healthy children. Clin Nephrol. 1979; 12(5):216-21.

21. Hogg RJ, Portman RJ, Milliner D, Lemley KV, Eddy A, Ingelfinger J. Evaluation and Management of Proteinuria and Nephrotic Syndrome in Children: Recommendations From a Pediatric Nephrology Panel Established at the National Kidney Foundation Conference on Proteinuria, Albuminuria, Risk, Assessment, Detection, and Elimination (PARADE). Pediatrics. 2000; 105(6):1242-1249.

22. Kidney Disease: Improving Global Outcomes (KDIGO) CKD Work Group. KDIGO 2012 Clinical Practice Guideline for the Evaluation and Management of Chronic Kidney Disease. Kidney inter Suppl. 2013; 3:1-150.

23. Piepsz A, Tondeur M. Revisiting normal $51 \mathrm{Cr}$ ethylenediaminetetraacetic acid clearance values in children. Eur J Nucl Med Mol Imaging. 2006; 33:1477-1482.

24. Aslam M, Watson AR. Unilateral multicystic dysplactic kidney: long term outcomes. Arch Dis Child. 2006; 91:820-823.

25. Rule AD, Glassock RJ. Classification of CKD should be about more than prognosis. Nat Rev Nephrol. 2013; 9:697-698.

Recibido: 10/02/2018

Aceptado: 18/09/2019 